\title{
A case of chylothorax due to diffuse large B-Cell lymphoma
}

\author{
Füsun Şahin* \\ University of Health Sciences, Yedikule Chest Diseases and Thoracic Surgery Training and Research Hospital, Turkey
}

\begin{abstract}
A 66-year-old male patient with was admitted to hospital with complaints of fatigue and shortness of breath. In the chest X-ray of the patient, homogeneous density increase was observed in the lower zone of the right lung, suggesting pleural effusion and showing Damoiseau's line. In the patient who underwent thoracentesis, pleural fluid in the form of yellow pus and empyema was seen. The pleural fluid biochemistry was in the character of exudate. Thoracic computed tomography (CT) was performed in the patient who underwent closed underwater drainage with tube thoracostomy. Pleural effusion was reaching $8 \mathrm{~cm}$. in the right hemithorax. The size of the spleen was increased, the liver parenchyma was heterogeneous. In the follow-ups, although the pleural effusion and Damoiseau line of the patient were erased, the fluid continued to come out of the drain as a daily empyema; the patient's clinical and laboratory tests were not compatible with empyema. In addition, a possible hematological malignancy was considered due to low $\mathrm{Hb}$, leukocyte, platelet levels, and hepatosplenomegaly. Chylothorax was taken into the differential diagnosis and triglyceride and cholesterol were sent from the pleural fluid. Triglyceride was high in pleural fluid. No malignant cells were seen in pleural fluid cytology. Positron emission tomography (PET)/CT was performed to determine the etiology in the patient who was diagnosed with chylothorax. There were dense hypermetabolic lymphadenopathy masses starting from the level of the 8th thoracic vertebra up to the retrocrural area in the prevertebral area. Spleen sizes were increased and there were intense hypermetabolic involvements. Involvement due to lymphoproliferative diseases was considered in the foreground. Mediastinoscopy was performed. Biopsies were taken from mass in the paraesophageal area (posterior mediastinum). The pathology result of the biopsies was "Diffuse Large B-Cell Lymphoma". The patient was referred to hematology and oncology clinics for treatment and discharged.
\end{abstract}

\section{Introduction}

Chylothorax is a rare condition that occurs when lymphatic fluid passes into the pleural space as a result of injury to the thoracic duct or compression of the lymphatic pathways [1]. The milk-like appearance of the formed effusion is characteristic. The fluids with triglyceride level above $110 \mathrm{mg} / \mathrm{dl}$ are defined as chylous pleural effusion. When the triglyceride level is below $50 \mathrm{mg} / \mathrm{dl}$, the diagnosis is excluded. When it is between $50-110 \mathrm{mg} / \mathrm{dl}$, lipid electrophoresis is required to show chylomicrons. Chylothorax formation as a result of interruption of the thoracic duct and lymphatic drainage may be due to traumatic or nontraumatic causes. While thoracic surgery is the main cause of traumatic chylothorax, malignancy is typically the most common cause of nontraumatic chylothorax. Nontraumatic chylothorax is divided into two groups as malignant and non-malignant. Malignancy-associated chylothorax is not uncommon [2,3]. Malignant chylothorax represents more than $50 \%$ of the cases of non-traumatic chylothorax, with most of them being caused by lymphoproliferative disorders [4]. In this article, a case of empyema-like chylothorax secondary to diffuse large B-cell lymphoma is presented.

\section{Case presentation}

A 66-year-old male patient with known diabetes mellitus and ischemic heart disease applied to our clinic with complaints of fatigue and shortness of breath. He was using metformin $1000 \mathrm{mg}$ for diabetes mellitus and ecoprin $100 \mathrm{mg}$ for ischemic heart disease. The patient, who underwent 2 stents with coronary angiography 1 year ago, had quit smoking 5 years ago and had a history of smoking 20 packet/year. There was no feature in his family history. In her physical examination, Blood Pressure Arterial: 110/70 mmHg, Pulse: 90/min, room air oxygen saturation was $97 \%$. There were no palpable lymph nodes in the neck and axilla. On auscultation, respiratory sounds were decreased in the right lower zone. On the postero-anterior radiograph of the patient, homogeneous density increase in the right lung, suggesting a pleural effusion and showing Damoiseau's line, was observed (Figure 1). In the hemogram and biochemistry of the patient, Albumin 45.8, Total protein: 69, LDH: 166, CRP:10.4, Creatinine: 1.40, Calcium: 11.7, LDH: 166, BUN: 96, WBC: 2.34, Hemoglobin: 9, Platelet: 100,000. In the patient who underwent thoracentesis, pleural fluid in the form of yellow pus and empyema was seen. Glucose: 264, Protein: 38, Albumin: 29, Adenosine Deaminase (ADA): 15, Pleural fluid $\mathrm{pH}$ : 7.56, LDH: 499 in pleural fluid. Computed tomography (CT) was performed on the patient who underwent closed underwater drainage with tube thoracostomy. In the thorax CT, pleural effusion reaching $8 \mathrm{~cm}$ in thickness between the pleural leaves in the right hemithorax and diffuse passive atelectasis areas were observed. (Figure 2) In the abdominal sections entering the study area; The size of the spleen was increased (splenomegaly), the liver parenchyma was heterogeneous, there was an $18 \mathrm{~mm}$ stone in the gallbladder lumen with a lobulated contour. In the follow-ups, despite the disappearance of pleural effusion and Damoiseau's line in the PA chest X-ray of the patient, fluid in the character of daily empyema continued to flow from the drain; the patient's clinical and laboratory tests are not compatible

${ }^{\star}$ Correspondence to: Füsun Şahin, Department of Pulmonology, University of Health Sciences, Yedikule Chest Diseases and Thoracic Surgery Training and Research Hospital, Zeytinburnu-İstanbul, Turkey, Tel: 009053271128 93, E-mail: fusunsahin19700@hotmail.com

Key words: chylothorax; lymphoma; pleural effusion

Received: May 27, 2021; Accepted: June 15, 2021; Published: June 18, 2021 


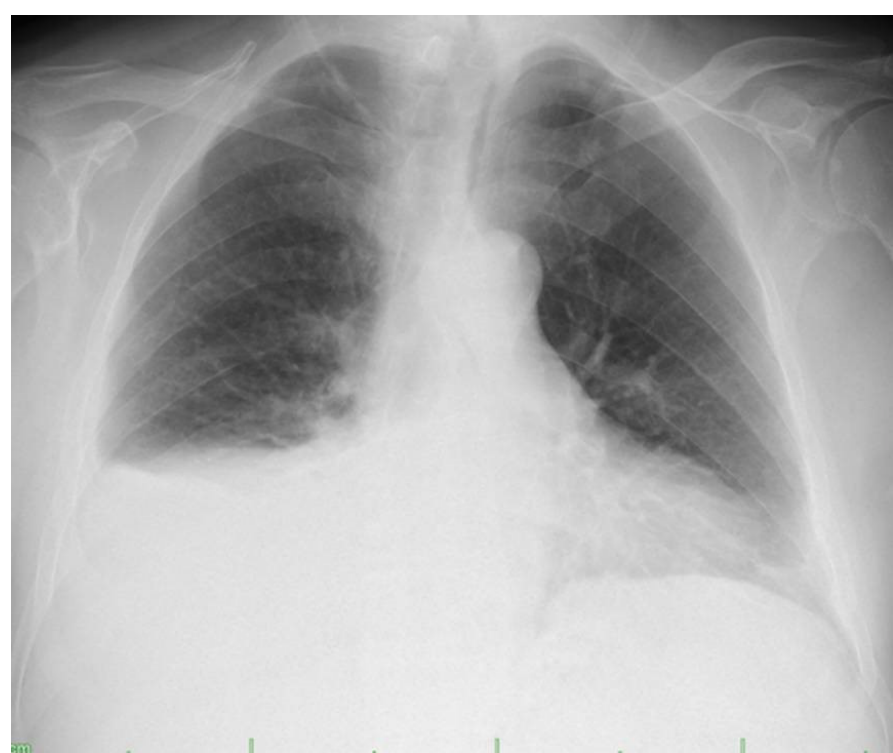

Figure 1: Postero-anterior (PA) chest radiograph of the patient. Homogeneous increase in density showing Damoiseau's line, suggesting pleural effusion in the lower zone of the right lung.

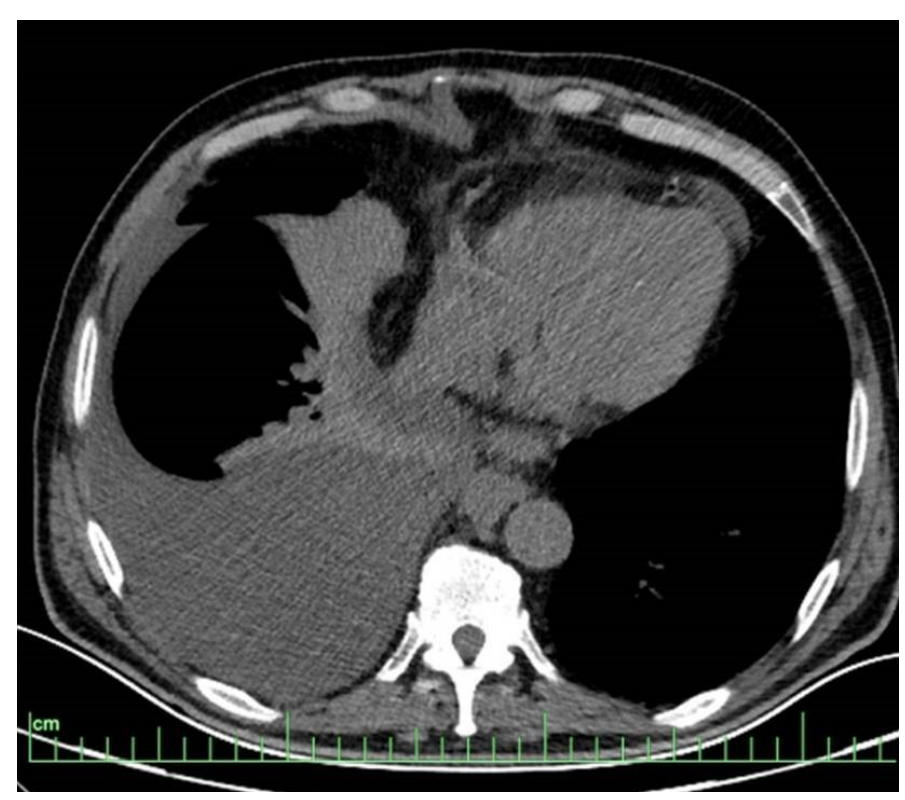

Figure 2: Pleural effusion reaching $8 \mathrm{~cm}$ in thickness between the pleural leaves in the right hemithorax on thorax $\mathrm{CT}$ and diffuse passive atelectasis areas adjacent to it.

with empyema. In addition, a possible hematological malignancy was considered due to low hemoglobin, leukocytes and platelets in the hemogram and hepatosplenomegaly. Chylothorax was taken into the differential diagnosis and triglyceride and cholesterol were sent from the pleural fluid. Total cholesterol in pleural fluid was 26 and triglyceride was 216. Collagen and vasculitis markers were negative, and no malignant cells were seen in pleural fluid cytology. There was no growth in the culture of the patient and acid-resistant dyeing (for tuberculosis) was negative. Oral intake was discontinued and total parenteral nutrition was started in the patient who was diagnosed with chylothorax. Positron emission tomography (PET)/CT was performed to determine the etiology in the patient whose pleural fluid was clearly drained by tube drainage. In the thoracic sections of the patient, no lesions showing pathological FDG uptake were found in both lung parenchyma, and there were dense hypermetabolic Lymphadenopathy (LAP) masses extending in the vicinity of the aorta descending to the retrocrural area in the prevertebral area of about $10 \mathrm{~cm}$ starting from the level of the thoracic 8th vertebrae (Figure 3). In addition, intense hypermetabolic Lymphadenopathy were observed in the abdomen at the level of the kidneys, in the precaval localization, at the mesenteric level adjacent to the left adrenal gland corpus, and at the level of the spleen hilum. There were intense hypermetabolic lymphadenopathies extending to the right common iliac and right internal-external iliac areas and also observed in the right inguinal area. Spleen sizes were observed to be larger than normal, and there were heterogeneous intense hypermetabolic involvements in the spleen parenchyma (Figure 3). When evaluated together with all these findings, involvement due to lymphoproliferative diseases was considered in the foreground. The patient who was spoken to by the thoracic surgery department of our hospital was told that the lymph nodes in the right inguinalexternal iliac area were deeply located and could not be sampled. Mediastinoscopy was performed with video-assisted thoracic surgery (VATS). In the posterior mediastinum, $8 \mathrm{~cm}$ in the paraesophageal area. mass was palpated. Multiple punch biopsies were taken from the mass. The pathology result of the biopsies taken from the mediastinal mass was "Diffuse large B-cell lymphoma". The patient was referred to hematology and oncology clinics for treatment and was discharged.

\section{Discussion}

The majority of patients with chylothorax present with dyspnea caused by the mechanical effects of pleural fluid. They may also present with a feeling of heaviness in the chest, fatigue and weight loss. Fever and chest pain are rare because chylous fluid does not produce an inflammatory response [5]. Chylothorax was also found in our case who presented with the complaint of increasing dyspnea due to pleural effusion. The most common disease causing chylothorax is nonHodgkin lymphoma. The most common causes of chylothorax other than lymphoma are surgical or non-surgical trauma and metastatic cancers. Some other rare causes are lymphangioleiomyomatosis, cirrhosis, heart failure, mitral stenosis, tuberculosis and sarcoidosis [6]. In accordance with this situation, the etiology was found to be non-Hodgkin lymphoma (diffuse large B-cell lymphoma) in our case. Chylothorax, which is not due to traumatic causes, may occur as a result of extrinsic compression or direct invasion of the tumor to the thoracic duct, or as a result of obliteration of the lymphatics such as radiation therapy [7]. Another mechanism is cisterna chyli due to lymphoma, cancer or rarely infections, as well as lymphadenopathy and abdominal obstruction due to compression. Chylous ascites formation is followed by absorption of diaphragmatic lymphatics or formation of chylous pleural effusion as a result of congenital diaphragmatic disorder [6]. In lymphoma, chylothorax develops due to stretching and tearing of the duct due to tumor compression or invasion by the tumor [8]. Unilateral pleural effusion is seen in approximately $78 \%$ of patients with chylothorax, mostly in the right hemithorax (67\%). Thoracic duct injury or obstruction below the fifth thoracic vertebrae with pleural fluid in the right hemithorax above this level damage usually results in pleural fluid in the left hemithorax. However, bilateral pleural fluid can also form [9]. In our case, the chylothorax was on the right side because the lymphadenopathy compression was at the level of the 8th thoracic vertebrae. The first procedure to diagnose chylothorax is thoracentesis. Although pleural fluid that is frequently taken is milk-like, the fluid may not be milky when the patient is malnourished, fasting for a long time, or on a low-fat diet. It can also appear as serous, serousangina or empyema. Chylous effusions are odorless, have exudate character and lymphocyte dominance in the fluid [10]. A triglyceride level higher than 

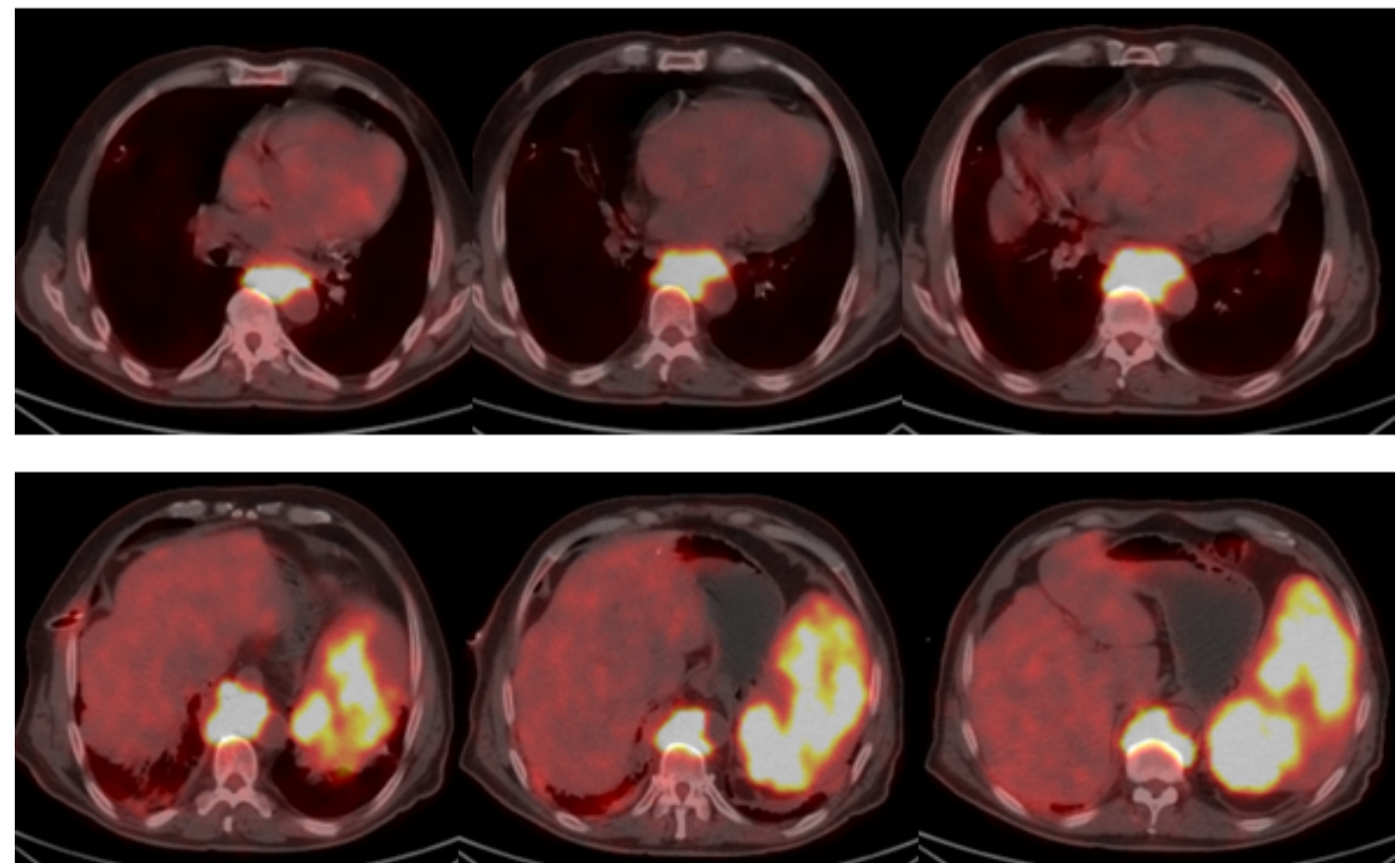

Figure 3: In PET/CT, no lesion showing pathological FDG uptake was detected in both lung parenchyma in thoracic sections. There were dense hypermetabolic lymph node masses starting from the T8 vertebra level, descending to the retrocrural area in the prevertebral area of approximately $10 \mathrm{~cm}$, and extending adjacent to the aorta. Spleen sizes were observed to be larger than normal, and there were heterogeneous intense hypermetabolic involvements in the spleen parenchyma.

$110 \mathrm{mg} / \mathrm{dl}$ in the pleural fluid establishes the diagnosis of chylothorax, while when it is between $50-110 \mathrm{mg} / \mathrm{dl}$, lipid electrophoresis is required to show chylomicrons [11]. If the drainage is below $1000 \mathrm{cc}$ per day, it is called low-volume, and if it is over $1000 \mathrm{cc}$, it is called chylothorax with high-volume leakage. The most important step in the treatment of chylothorax is the detection of the underlying disease. In some cases, such as the steroid treatment given in sarcoidosis or the treatment of heart failure, the chylothorax will disappear with treatment [12]. Patients who do not need emergency surgery are those with lowvolume leaks or postoperative patients with high-volume leaks that respond significantly to conservative treatment. In most cases, the underlying disease should be treated, dietary changes, and pleural drainage should be performed for symptom control [13]. Pleural drainage should be provided with the aid of a chest tube or indwelling catheter for symptom control. Low-fat and high-protein foods should be preferred in the diet. This strategy reduces the rate of accumulation of chylous fluid in many patients and, in some cases, allows it to heal spontaneously without the need for surgery. Apart from conservative and surgical approaches, it is necessary to review alternative methods in the treatment of chylothorax. Among these alternative treatments are pleurodesis with talc or povidoneiodine or the recently popular OK-432 (picibacilli), sclerotherapy to dilated lymphatics with tetracycline, fluoroscopic embolization of the ductus thoracic duct with platinum coils, and pleuroperitoneal shunt and pleurectomy [14,15]. Although the treatment of the disease gives good results, especially in many diseases such as malignant lymphoma, chylothorax may recur and therefore additional precautions must be taken. In these patients, especially when chylous drainage of 3 liter or more per day is provided, significant metabolic and nutritional problems may develop due to severe protein and vitamin loss. Therefore, the most important stage of conservative treatment is the monitoring and regulation of nutrition and fluid electrolyte balance. Interruption of oral intake together with nutritional formulas containing medium chain triglycerides and total parenteral nutrition is the first treatment to be applied [8]. Our case was evaluated as chylothorax with high volume leakage and not requiring emergency surgery. For symptom control, closed underwater drainage with tube thoracostomy was performed and diet was regulated. However, fluid drainage could not be reduced with conservative treatments. When the etiology of chylothorax was found to be diffuse large B-cell lymphoma in our patient, the patient was referred to the oncology clinic and the underlying disease was treated.

\section{Conclusion}

After detecting chylothorax, the first step is to find the disease causing the chylothorax. It should be kept in mind that lymphoma may cause chylothorax, as in our patient, a detailed anamnesis should be taken from the patients and a physical examination should be performed. Intrathoracic and intraabdominal lymph nodes should be evaluated by performing advanced examinations such as thorax and abdomen CT, PET/CT. Conservative treatments together with the treatment of the underlying disease also contribute to the improvement of the disease.

\section{References}

1. Gomes AO, Ribiero S, Neves J, Mendonça T (2015) Uncommon aetiologies of chylotoraks: superior vena cava syndrome and thoracic aortic aneurysm. Clin Respir $J$ 9: 185-188. [Crossref]

2. Doerr CH, Allen MS, Nichols FC 3rd, Ryu JH (2005) Etiology of chylothorax in 203 patients. Mayo Clin Proc 80: 867-870. [Crossref]

3. Kako S, Joshita S, Matsuo A, Kawaguchi K, Umemura T, et al. (2019) A Case of Adult T-Cell Leukemia/Lymphoma Complicated with Bilateral Chylothorax. Case Rep Oncol Med 2019: 8357893. [Crossref]

4. Pospiskova J, Smolej L, Belada D, Simkovic M, Motyckova M, et al. (2019) Experiences in the treatment of refractory chylothorax associated with lymphoproliferative disorders. Orphanet J Rare Dis 14: 9.

5. Prakash UBS (2002) Chylothorax and pseudochylothorax. Eur Respir Mon 7: 249.

6. Valentine VG, Raffin TA (1992) The management of chylothorax. Chest 102: 586-591. 
7. Sassoon CS, Light RW (1985) Chylothorax and pseudochylothorax. Clin Chest Med 6: 163-171. [Crossref]

8. Şahin E, Çelik B, Nadir A (2011) A case of non-Hodgkin lymphoma presenting with tension chylothorax. Turkish Thoracic Cardiovascular Surgery 19: 660-663.

9. Miller JI. Anatomy of the thoracic duct and chylothorax. In Shields TW, LoCicero J, Ponn RB, Rusch VW. ed. General thoracic Surgery $\left(6^{\text {th }}\right.$ edn $)$. Philadelphia: Lippincott Williams and Wilkins; 2005; 1: 879-88.

10. Huggins JT (2010) Chylothorax and cholesterol pleural effusion. Semin Respir Crit Care Med 31: 743-750. [Crossref]

11. Maldonado F, Hawkins FJ, Daniels CE, Doerr CH, Decker PA, et al. (2009) Pleural fluid characteristics of chylothorax. Mayo Clin Proc 84: 129-133. [Crossref]
12. Hillerdal G (1997) Chylothorax and pseudochylothorax. Eur Respir J 10: 1157-1162. [Crossref]

13. Densupsoontorn NS, Jirapinyo P, Wongarn R, Thamonsiri N, Nana A, et al. (2005) Management of chylothorax and chylopericardium in pediatric patients: experiences at Siriraj Hospital, Bangkok. Asia Pac J Clin Nutr 14: 182-187. [Crossref]

14. Marcon F, Irani K, Aquino T, Saunders JK, Gouge TH, et al. (2011) Percutaneous treatment of thoracic duct injuries. Surg Endosc 25: 2844-2848. [Crossref]

15. Lim KA, Kim SH, Huh J, Kang IS, Lee HJ, et al. (2005) Somatostatin for postoperative chylothorax after surgery for children with congenital heart disease. J Korean Med Sci 20: 947-951. [Crossref]

Copyright: (C2021 Şahin F. This is an open-access article distributed under the terms of the Creative Commons Attribution License, which permits unrestricted use, distribution, and reproduction in any medium, provided the original author and source are credited. 